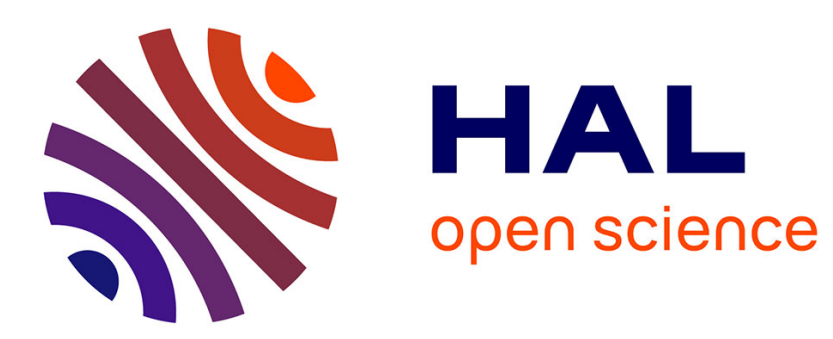

\title{
Feigenbaum's ratios of two-dimensional area preserving maps
}

Bernard Derrida, Y. Pomeau

\section{To cite this version:}

Bernard Derrida, Y. Pomeau. Feigenbaum's ratios of two-dimensional area preserving maps. Physics Letters A, 1980, 80 (4), pp.217-219. 10.1016/0375-9601(80)90003-1 . hal-03285939

\section{HAL Id: hal-03285939 \\ https://hal.science/hal-03285939}

Submitted on 19 Jul 2021

HAL is a multi-disciplinary open access archive for the deposit and dissemination of scientific research documents, whether they are published or not. The documents may come from teaching and research institutions in France or abroad, or from public or private research centers.
L'archive ouverte pluridisciplinaire HAL, est destinée au dépôt et à la diffusion de documents scientifiques de niveau recherche, publiés ou non, émanant des établissements d'enseignement et de recherche français ou étrangers, des laboratoires publics ou privés. 


\title{
FEIGENBAUM'S RATIOS OF TWO-DIMENSIONAL AREA PRESERVING MAPS
}

\author{
B. DERRIDA and Y. POMEAU \\ CEN-Saclay, 91190 Gif-sur-Yvette, France
}

Received 25 September 1980

\begin{abstract}
We generalize simple renormalization methods to calculate Feigenbaum's ratios for a two-dimensional area preserving map. Our results are in satisfactory agreement with known results and give approximate estimate for the ratio associated to more general cascades like $1,3,3^{2}, \ldots, 3^{n}, \ldots$.
\end{abstract}

Maps of the interval have attracted a lot of attention in the last few years (some recent references can be found in ref. [1]). The interest of physicists for these mathematical models was increased by the work of Feigenbaum [2,3] who discovered that the perioddoubling cascades have universal critical properties: for a large class of one-dimensional maps $T_{\mu}$ which depend on a deformation parameter $\mu$, there is a cascade of bifurcations $1 \rightarrow 2 \rightarrow 4 \rightarrow \ldots \rightarrow 2^{n} \rightarrow \ldots$. The values $\mu_{n}$ of the parameter where the cycle $2^{n}$ becomes unstable and where a cycle $2^{n+1}$ appears accumulate at some value $\mu_{\infty}$. Feigenbaum pointed out that for a large class of maps $T_{\mu}$, one has

$$
\lim _{n \rightarrow \infty} \frac{\mu_{n}-\mu_{\infty}}{\mu_{n+1}-\mu_{\infty}}=\delta \text {, }
$$

$\delta$ is a universal number which depends only, on the shape of the map $T_{\mu}$ near its maximum. When the maximum of $T_{\mu}$ is quadratic, the value of $\delta$ is

$$
\delta=4.66920 \text {. }
$$

As mentioned by Eckmann [4], the same value of $\delta$ was obtained by several authors [5-7] when they studied other dissipative systems and it is believed [8] that this value will be soon measured in the cascades observed in Rayleigh Benard experiments.

A renormalization group theory of these perioddoubling cascades was proposed by Feigenbaum $[2,3]$ and rigorous results were derived by Collet et al. [9] . For maps like $\left(x \rightarrow 1-a|x|^{1+\epsilon}\right)$ it was possible to expand $\delta$ for small values of $\epsilon[7,9]$. In our work [7] with A. Gervois, we developed several approximate renormalization methods similar to real space renormalizations used in statistical physics. They led to rather accurate values of $\delta$. Moreover, in ref. [7], the period doubling phenomenon was generalized to other cascades like $1,3,3^{2}, \ldots, 3^{n}, \ldots$. Though for these new cascades, the stability zones of the cycles (values of $\mu$ where the cycle is stable) are not contiguous, well defined periods $3^{n}$ appear at values $\mu_{n}^{\prime}$ which accumulate to $\mu_{\infty}^{\prime}$ in the same way as in period-doubling cascades:

$\lim _{n \rightarrow \infty} \frac{\mu_{n}^{\prime}-\mu_{\infty}^{\prime}}{\mu_{n+1}^{\prime}-\mu_{\infty}^{\prime}}=\delta^{\prime}$.

The number $\delta^{\prime}$ is also universal and characteristic of the factor 3 . For maps with a quadratic maximum, its value is

$\delta^{\prime}=55.247 \ldots$.

Again this universal ratio can be recovered approximatively by simple renormalization equations.

In this letter, we use the same kind of renormalization equations to calculate the ratios $\delta$ and $\delta^{\prime}$ for twodimensional area preserving maps. The importance of these maps is that they are good examples of conservative dynamical systems. We generalize to the two-dimensional case the method called "equality of slopes" in ref. [7]. The principle of the method is the following: consider a two-dimensional map $(x, y) \rightarrow T_{\mu}(x, y)$. Here we take for $T_{\mu}$ the Hénon map in the area preserving case [10] 
$T_{\mu}(x, y)=\left(1-\mu x^{2}+y,-x\right)$

For the simplest cycles (cycles of length $1,2,3,4$ ) one can calculate analytically the coordinates of the points of a cycle as functions of $\mu$. We can also linearize the map $T_{\mu}^{(n)}$ in the neighbourhood of the points of cycle $n$. This linearized map is a $2 \times 2$ matrix $M$ given by

$M=\prod_{i=1}^{n}\left(\begin{array}{cc}-2 \mu x_{i} & 1 \\ -1 & 0\end{array}\right)$,

where the $x_{i}$ are the coordinates of the points of cycle $n$. The eigenvalues $\lambda_{n}$ of this matrix can be expressed as a function of $\mu$. For $n=1,2,3,4$, the $\lambda_{n}$ are roots of

$\lambda_{n}^{2}+2 f_{n}(\mu) \lambda_{n}+1=0$

with

$f_{1}(\mu)=(1+\mu)^{1 / 2}-1, \quad f_{2}(\mu)=2 \mu-7$,

$f_{3}(\mu)=4 \mu\left[1+(\mu-1)^{1 / 2}\right]-5-3(\mu-1)^{1 / 2}$,

$f_{4}(\mu)=8 \mu^{2}-16 \mu^{3 / 2}-1$.

(Note that the cycle $n$ is elliptic if $-1 \leqslant f_{n}(\mu) \leqslant 1$.)

For period doubling problems, the idea of renormalization is to try to associate at each value of $\mu$, a value $\mu^{\prime}$ such that $T_{\mu}$ looks like $T_{\mu}^{(2)}$. An approximate way to do so is to say that the linearization of $T_{\mu}^{(n)}$ around a point of cycle $n$ is identical to the linearization of $T_{\mu^{\prime}}^{(2 n)}$ around a point of cycle $2 n$. Therefore:

$f_{n}(\mu)=f_{2 n}\left(\mu^{\prime}\right)$.

This relation provides an approximate value for $\mu_{\infty}$ (the value of $\mu$ where the cascade $1 \rightarrow 2 \rightarrow \ldots \rightarrow 2^{n} \ldots$ accumulates) and of $\delta . \mu_{\infty}$ is the fixed point of renormalization (8)

$f_{n}\left(\mu_{\infty}\right)=f_{2 n}\left(\mu_{\infty}\right)$,

whereas $\delta$ is given by:

$\delta=\mathrm{d} \mu /\left.\mathrm{d} \mu^{\prime}\right|_{\mu_{\infty}}$.

The choice of small value of $n$ constitutes an approximation which should be improved by increasing $n$. We have done the calculations for $n=1$ and $n=2$ and the results are summarized in table 1 . In the same way, one can study the cascade $1,3,3^{2}, \ldots, 3^{n} \ldots$ which accumulates at value $\mu_{\infty}^{\prime}$ with a Feigenbaum ratio $\delta^{\prime}$. The re-
Table 1

Results of the approximate renormalizations for the Henon map $(x, y) \rightarrow\left(1-\mu x^{2}+y,-x\right)$. The expected values are unknown except $\delta$ which has been recently obtained by several authors $[11-15]$.

\begin{tabular}{llll}
$n$ & $2 n$ & $\mu_{\infty}$ & $\delta$ \\
1 & 2 & 4.13278 & 9.0623 \\
2 & 4 & 4.13621 & 8.6845 \\
expected values & $?$ & $8.72 \ldots$ \\
$n$ & $3 n$ & $\mu_{\infty}^{\prime}$ & $\delta^{\prime}$ \\
\hline 1 & 3 & 1.18362 & 22.869 \\
expected values & $?$ & $?$
\end{tabular}

normalization equation ( 8 ) becomes in this case:

$f_{n}(\mu)=f_{3 n}\left(\mu^{\prime}\right)$,

and the procedure remains the same to find $\mu_{\infty}^{\prime}$ and $\delta^{\prime}$. We have done in this case the calculations for $n=1$ and the results are also given in table 1 . They agree rather well with recent works [11-15] for the period doubling ratio $\delta$ and provide an estimate for the period tripling ratio $\delta^{\prime}$.

In order to show the accuracy of these calculations, we reproduce in table 2 the results obtained [7] with the same method for the well known one-dimensional map $T_{\mu}\left(x \rightarrow 1-\mu x^{2}\right)$. In this case the method is not very different from the one used by May and Oster [1] to calculate approximately $\delta$.

The method we used in this letter gives rather accurate results with a very small amount of calculations. From tables 1 and 2 , there is no doubt that the universal ratios $\delta$ and $\delta^{\prime}$ are different for conservative systems (like the Hénon map) and dissipative systems (like $x$

Table 2

Results of the approximate renormalization for the one-dimensional map $x \rightarrow 1-\mu x^{2}$.

\begin{tabular}{llll}
\hline$n$ & $2 n$ & $\mu_{\infty}$ & $\delta$ \\
\hline 1 & 2 & 1.39039 & 5.123 \\
2 & 4 & 1.40142 & 4.614 \\
expected values & 1.40115519 & 4.66920 \\
$n$ & $3 n$ & $\mu_{\infty}^{\prime}$ & $\delta^{\prime}$ \\
\hline 1 & 3 & 1.78597 & 61.61 \\
expected values & 1.786440255 & 55.247
\end{tabular}


$\left.\rightarrow 1-\mu x^{2}\right)$. It would be interesting to know more about the ratios $\delta$ one can define for cascades like $p$, $p^{2}, p^{3}, \ldots, p^{n}, \ldots$ and to see if the $p$ dependence of these universal ratios is simple. Finally, it seems more difficult in the conservative case to define a map (like $x \rightarrow 1-a|x|^{1+\epsilon}$ in the dissipative case) where an analytic expansion of $\delta$ and $\delta^{\prime}$ can be done.

We want to thank especially Professor J.P. Eckmann for several discussions on this problem and to acknowledge Professor J. Ford and Professor R. MacKay for communicating us their results. After completion of this work, we have been informed by R.H.G. Helleman [16] of a R.G. calculation of $\delta$ in the period doubling case giving the same result as ours.

\section{References}

[1] R.M. May and G.F. Oster, Phys. Lett. 78A (1980) 1.

[2] M.J. Feigenbaum, J. Stat. Phys. 19 (1978) 25.
[3] M.J. Feigenbaum, J. Stat. Phys. 21 (1979) 669.

[4] J.P. Eckmann, in: Bifurcation phenomena in mathematical phy sics and related topics, eds. C. Bardos and D. Bessis (Reidel, 1980) pp. 115-135.

[5] V. Franceschini, J. Stat. Phys. 22 (1980) 397.

[6] V. Franceschini and C. Tebaldi, J. Stat. Phys. 21 (1979) 707.

[7] B. Derrida, A. Gervois and Y. Pomeau, J. Phys. A12 (1979) 269.

[8] M.J. Feigenbaum, Phys. Lett. 74A (1979) 375.

[9] P. Collet, J.P. Eckmann and O.E. Landford, preprint HUTMP 79/B85.

[10] M. Hénon, Quart. Appl. Math. 27 (1969) 291; Commun. Math. Phys. 50 (1976) 69.

[11] G. Bennetin, C. Cercignani, L. Galgani and A. Giorgilli, Lett. Nuovo Cimento 28 (1980) 1.

[12] J.P. Eckmann, private communication.

[13] J.M. Green and R. MacKay, to be published.

[14] J. Ford and F. Vivaldi, to be published.

[15] T. Bountis, to be published.

[16] R.H.G. Helleman, Fundamental problems in statistical mechanics, Vol. 5, ed. E.G.D. Cohen (North-Holland, 1980), to be published. 